\title{
2021 Elijah High-Altitude Balloon Payload
}

\author{
Jordan Cioni ${ }^{1}$, Jorge Gutierrez ${ }^{2}$, Julia Kalish ${ }^{2}$, Bryanna Salazar ${ }^{2}$ \\ University of Wisconsin-Green Bay ${ }^{1}$, Milwaukee School of Engineering ${ }^{2}$
}

\begin{abstract}
The 2021 WSGC Elijah High-Altitude Balloon Payload Team focused their projects on the upcoming 2024 solar eclipse. These included solar sensing, solar imaging, and the study of cosmic radiation. The solar sensing project involved collecting flight data such as altitude, temperature, and pressure. In addition to this data, quantitative UVA, UVB, light intensity, and solar energy values using various sensors and solar panels. Solar imaging utilized an action camera with solar, IR, and IR cut-off filters in conjunction with a 360-degree camera that captured the entire payload's flight. The radiation shielding project focused on studying the effectiveness of low-density polyethylene as a radiation shielding material by comparing observations collected by shielded and unshielded Geiger counters. To observe radiation originating from nearby star systems, a particle detector consisting of a scintillator coupled to a silicon photomultiplier was constructed.
\end{abstract}

\section{Overview}

The Elijah High-Altitude Balloon Program is an undergraduate research program established by the Wisconsin Space Grant Consortium. Its purpose is to equip students with valuable teamwork experience and exposure to aerospace-related research. The 2021 team continued with experiments that are similar to experiments performed by the previous year's team to refine specific experiments planned for the solar eclipse in 2024. Research topics explored included: solar sensing, solar imaging, shielding from galactic cosmic radiation, and the interstellar radiation landscape. A unique focus was capturing footage of the entire flight and was made possible by utilizing a 360-degree camera. This paper will describe the launch day weather conditions and discuss the payload's overall construction. Additionally, background information, experimental methodology, and results will be provided for the solar sensing, solar imaging, radiation shielding, and radiation landscape studies.

\section{Launch Day Weather Conditions}

Weather conditions on launch day comprised of temperatures reaching $92^{\circ} \mathrm{F}$, sunny with some clouds, and wind speeds reaching $21 \mathrm{mph}$. There was no precipitation during launch or flight.

This material is based upon work supported by NASA under Award No. EBP21_4.0 issued through the Wisconsin Space Grant Consortium. Any opinions, findings, and conclusions or recommendations expressed in this material are those of the author(s) and do not necessarily reflect the views of the National Aeronautics and Space Administration. 


\section{Payload Design}

Introduction Design parameters for the payload focused on weight, accessibility to sensors, ease of use, and video capture. Once the team considered FAA regulations, it was clear that the payload could not exceed six pounds. The payload design resembled a cylinder, as previous experiments have demonstrated its low weight and durability.

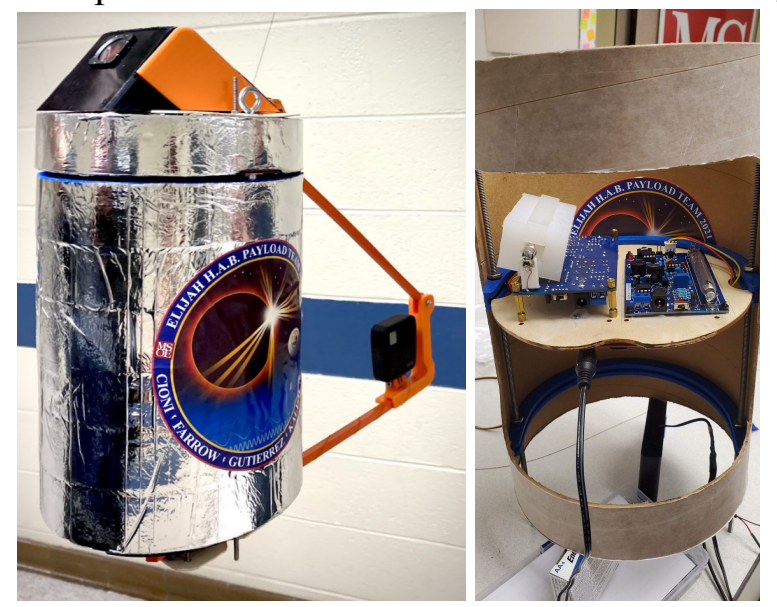

Figure 1. The image above is the complete assembled payload with each experiment attached in their designated locations.

Methods Materials on the payload included insulation foam, a rocket body tube, 3D printed support structures, plywood, and hardware. The team used a hot copper wire cutter to cut the insulation foam into cylindrical shapes with a hole in the middle. The rings were stacked and glued together to form the outer part of the payload. The team adhered a Mylar blanket to the outside of the foam rings for added insulation. The team took inspiration for the shelf design from the 2019 Payload Team (Beaty, Endres, Hennigan, Shuler, Washburn, \& Wolfgramm, 2019). 3D printing and laser cutting were methods used to fabricate the shelf slots and the shelves (Figure 1). Guide rings were 3D printed and added to the inside of the insulation foam rings to ensure a tight and secure fit between the rocket body tube and the surrounding shell.

Results The payload remained completely intact upon retrieval. There was no evidence to suggest that there was any damage to the structure. Therefore, there is no evidence to conclude that any structural damage would impact data. Some means of attachment secured each experiment to limit movement. For example, heavy-duty velcro firmly secured the battery packs on the bottom layer of the payload. Similarly, velcro secured the rest of the experiments within the payload body. The outside experimental parts were screwed into the payload body using plywood and suitable nuts for each screw.

Conclusion The design was successful overall in protecting the experiments inside and out. In taking inspiration from the 2019 team's shelving system, we successfully implemented a cleaner and sturdier strategy for ensuring the physical safety of each experiment. Using velcro improved the overall structure because it was easier to alter the location of individual components. Future flight observations will contribute to this payload design and ensure effectiveness in keeping contents safe and secure during flight. 


\section{Solar Sensing}

Introduction There are key elements for any flight in order to collect and organize data efficiently and allow for easy comparison between experiment data. The basic data collected on this flight included tracking the altitude, temperature, pressure, and time throughout the duration of the flight. This information is crucial to use when comparing other data from the flight and allows for easy reference when analyzing all the data. An example of this would highlight specific details of the flight such as when and at what altitude an analyzed event occurred. Hence why an altimeter and a time-keeping sensor (real-time clock - RTC) were vital to consider when purchasing multiple sensors. It was decided that collecting light intensity values, not only in general, but on various parts of the payload were vital to compare data obscured by the payload itself. By collecting light intensity data directed onto the payload, this would allow for a more accurate analysis of how much light was under experimentation for other experiments. An ambient light sensor and two solar panels determined the approximate light intensity and amount of solar energy produced throughout the flight. These sensors and panels collected quantitative data to compare to data collected during the 2024 solar eclipse flight. Based on the interest of collecting data to investigate the sun's corona, specific sensors were needed. This part of the sun is above the outermost atmosphere and is the hottest aspect of the sun, even hotter than the surface which reaches approximately 5,500 degrees Celsius. Ultraviolet light is known to map and trace the tenuous plasma that makes up the sun's corona; this fact led to the purchase and use of an ultraviolet sensor.

Methods The sensors chosen for the flight were QWIIC SparkFun Sensors. These sensors utilize the Inter-Integrated Circuit (IIC) Protocol to connect boards and sensors. The IIC Protocol allows the chips in multiple digital integrated circuits to interface and communicate with various other chips for control commands. These sensors were chosen due to the simplicity of prototyping with an IIC interface. The QWIIC connect system also uses 4-pin JST connectors to interface more rapidly. The ultraviolet sensor used was the VEML6075 (QWIIC) sensor from SparkFun. The ambient light sensor was the VEML6030 (QWIIC) sensor from SparkFun as well. On the other hand, the altimeter (MPL3115A2) did not have the IIC interface. A SparkFun QWIIC Adapter allowed for the fast and easy connection between the altimeter and other sensors. A SparkFun QWIIC real-time clock module (RV8803) recorded times at which events occurred for easy comparison between data relevant to specific points during the flight. QWIIC connectors made communication between the microcontroller and each sensor more efficient. A SparkFun RedBoard microcontroller and a QWIIC Shield allowed for multiple QWIIC connections to the same board. Figure 2 shows the female wires soldered to the QWIIC Shield, allowing the solar panels to be easily disconnected and reconnected to the board. A SparkFun QWIIC OpenLog micro SD card reader was used and mounted onto the QWIIC Shield to store data collected from each sensor. 


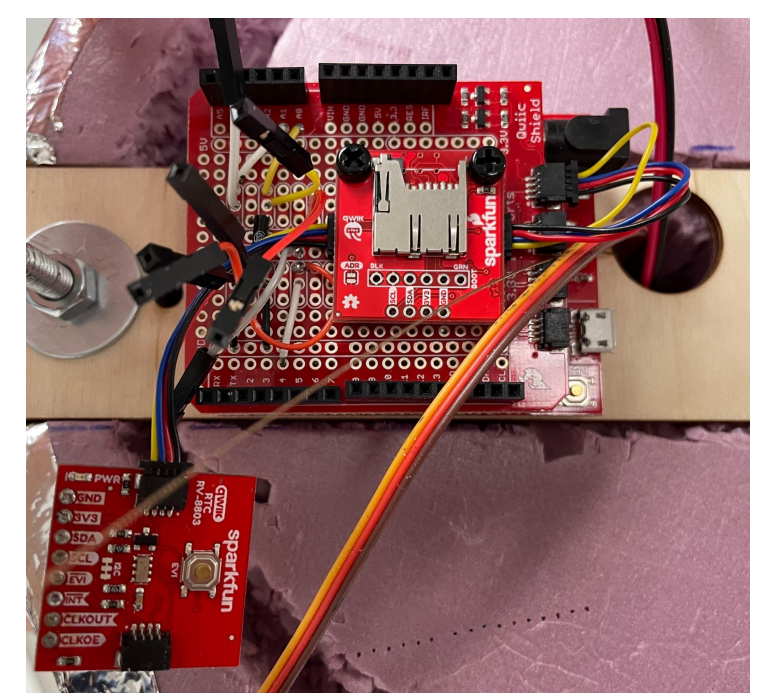

Figure 2. RedBoard microcontroller with soldered female connectors along with the OpenLog micro SD card reader connected to the RTC module.

The altimeter, ultraviolet sensor, and ambient sensor were each mounted onto the top of the lid of the payload in a PLA 3D printed casing (Figure 3).

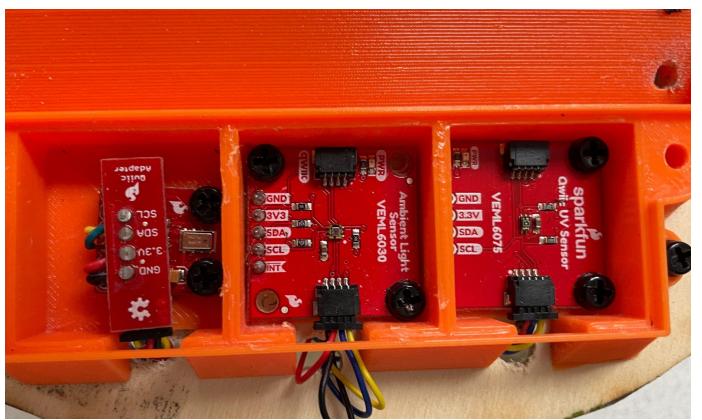

Figure 3. From left to right: An altimeter, ultraviolet light sensor, and ambient light sensor are mounted in a PLA casing with QWIIC connectors attached.

Results The flight data collected by the altimeter proved to align with previous expectations mostly. When plotted, the pressure and temperature acted as expected, decreasing as the altitude increased, thus seeing that the minimum of those graphs would indicate the point at which the balloon burst. On the other hand, the altitude data portrayed the payload as if it were going underground. It is unknown what the error was for the altimeter data. The connections and placement of the sensor was correct at the time of liftoff and the sensor was still intact after landing. In the future, it would be helpful to purchase and experiment with various brands of altimeter sensors and test each one separately. By doing this, the experimental and expected results of altitude based on the testing location should be the deciding factors for which sensor should be selected. The ground pressure was approximately $24,000 \mathrm{~m}$ and had a burst altitude of $399 \mathrm{~m}$. Despite this, the team was able to make approximations from observing the pressure data. The approximate burst time and altitude were 1:51 p.m. and 24,200 above sea level. 

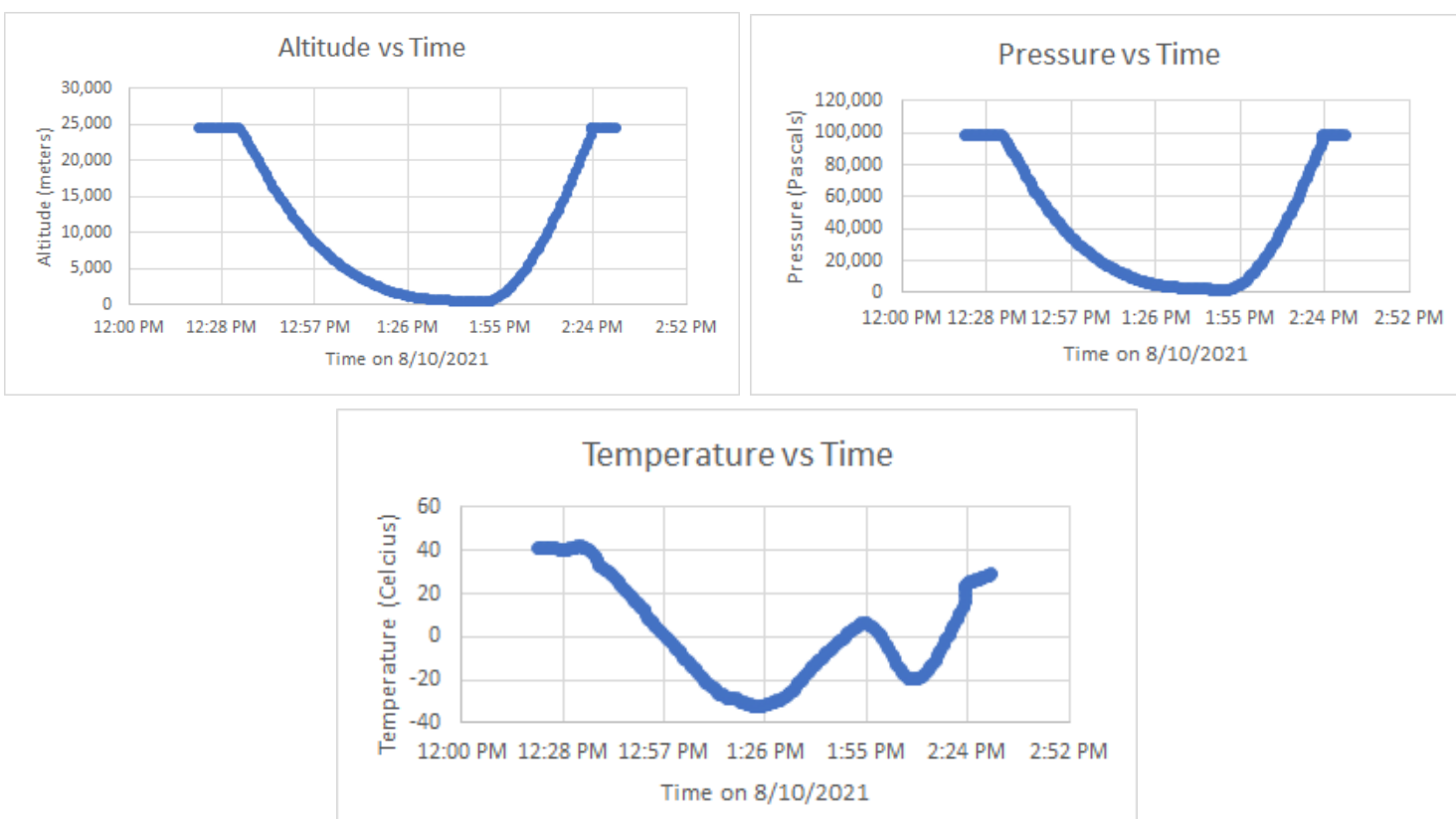

Figure 4. Graphs of the altitude, pressure, and temperature collected by the on-board altimeter and RTC.

The on-board altimeter recorded data represented in Figure 4 such as the altitude (in meters), pressure (in Pascals), and temperature (in Celsius). The data collected from the ambient, ultraviolet, and solar panels all acted as expected with increasing altitude. Figure 5 demonstrates how the ambient light was very high throughout the flight since it was a clear day with few clouds. It also shows that the UV radiation increased until the balloon burst and gradually decreased as the payload descended. The same observation holds for solar energy because energy was more intense until the balloon burst and went down in intensity during the payload's descent. The variability between the ambient light and UV radiation could have been due to slightly inaccurate data collection from the ambient light sensor due to the violent rocking of the payload throughout the flight. The sensitivity of the ambient light sensor may not have been proficient for the amount of light exposure the sensor received.
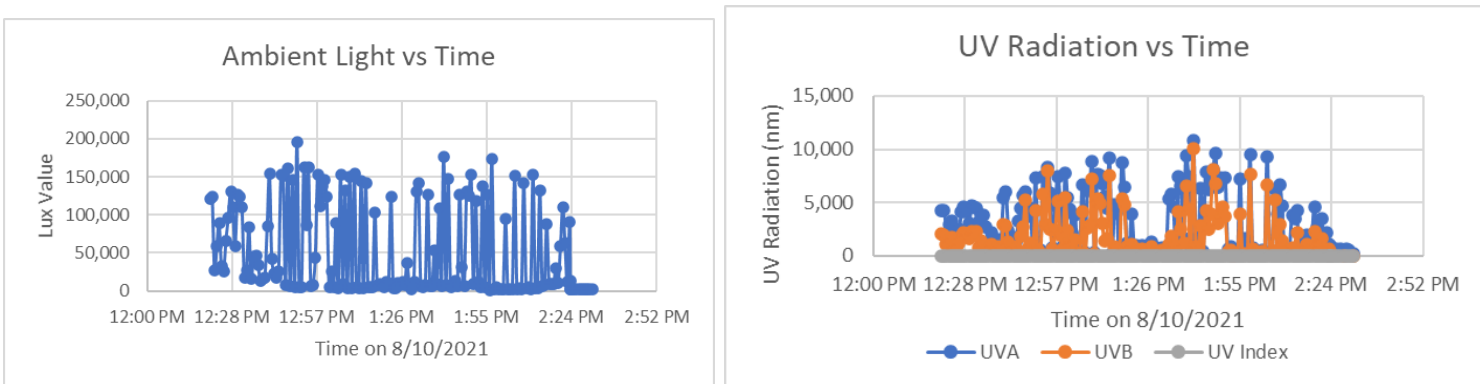


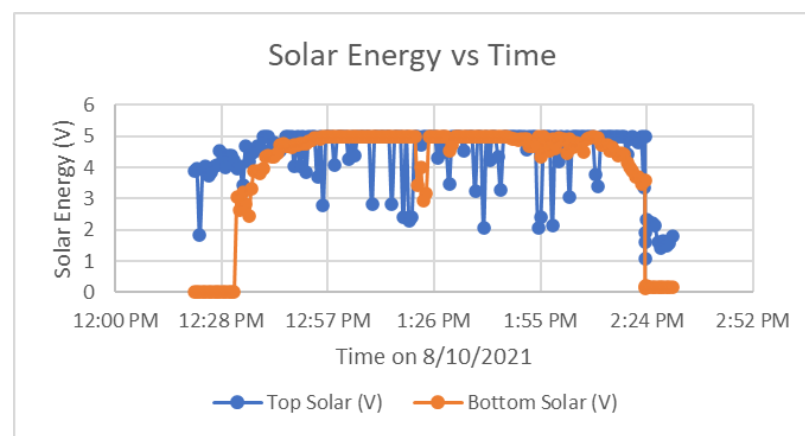

Figure 5. Graphs of the ambient, UV radiation, and solar energy values collected. In the Solar Energy vs Time graph, 'Top solar' refers to the solar panel placed on the top of the payload, the same goes for 'Bottom solar.'

Conclusion Despite the minor errors that occurred with the altitude collection, the rest of the sensors provided meaningful data to reference as control data for the upcoming 2024 solar eclipse flight. These sensors are very efficient due to their low power draw and negligible weight. The ultraviolet light data is meaningful when compared to the study of the corona during totality. When the eclipse reaches totality, the effects on solar energy and the atmosphere around the payload will become evident.

\section{Solar Imaging}

Introduction With the focus on the 2024 eclipse, recording the event was one of the team's objectives from the start, as a continuation of the previous year's team. The main objective was to capture and study the sun and its corona during the solar eclipse. Additionally, never before has the balloon payload been recorded in flight from the outside. A camera mounted outside the payload and facing it would give a new perspective on the flight and show how the payload behaves when the balloon bursts at maximum altitude. Due to budget and time limitations, we chose a full spectrum action camera (Akaso EK7000) and a 360-degree camera (GoPro Fusion). Three different filters were used with the full spectrum camera: a solar filter, an IR filter, and an IR cut-off filter. These filters rotated in front of the camera's lens. The full spectrum camera was used due to its ability to detect light more sensitively which has proven to be superior for astronomical imaging. Solar filters are used to photograph the sun during an eclipse by spectators, thus one was added to record what a normal person would see from Earth. The cut-off filter was added to make the full spectrum camera a typical consumer camera to compare with the other filtered video. The last filter implemented was the IR filter, which would allow the team to view the sun's infrared light and nothing else. The 360-degree camera would capture the sun in its raw form and provide a new perspective for the flight. A 360-degree camera records views from above and below, which will allow the payload itself to be visible during the flight. Figure 6 shows the two cameras and the filtering mechanism flown on the top lid. 


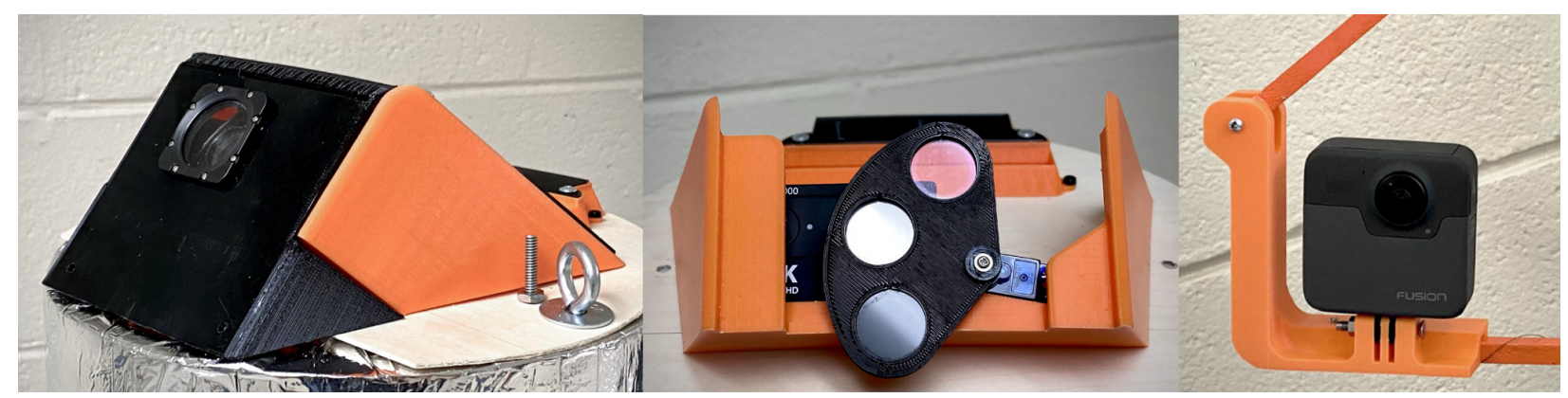

Figure 6. Left: Full spectrum camera enclosure. Middle: Filter mechanism. Right: 360 degree camera with selfie stick.

Methods To change the action camera into a full-spectrum camera, we removed the front cover, the glue from the lens threads, the lens, and the small glass infrared cut-off filter. The camera was then plugged into a computer and set up as a webcam for easy viewing. The process of reattaching the lens involved screwing it back on until the picture focused. After reattaching the cover, the full-spectrum camera was ready. The 360-degree camera used two 64 GB memory cards. Both cameras were left to record on fresh batteries until they died. With the battery capacity and length of time known, we could calculate the discharge rate. The flight time would be about two hours, so we determined that a 7500 mAh power bank would be enough to keep the cameras charged. With limited availability for two input port power banks, we selected a $13000 \mathrm{mAh}$ battery that weighed nine ounces.

The filter mechanism used a Tower Pro SG92R micro servo to rotate the filters. The servo connected to the Solar Sensing Arduino, and it was programmed to undergo a rotation every 10 seconds. OnShape was used to design the case that would hold the three $25 \mathrm{~mm}$ filters that would later be 3D printed using black PLA filament. The team utilized OnShape to create a virtual assembly of all the components to ensure they fit together without interference. The base at the top of the payload supported the full spectrum camera and filter mechanism to diminish any snagging with the payload train strings and parachute. We printed the base and its shield with black and orange PLA filament. The full spectrum camera was angled 45 degrees so that the balloon was visible in the top half of the camera frame. This angle was calculated using an aspect ratio of 16:9 and knowing that the lens had a maximum viewing angle of 170 degrees horizontally.

The selfie stick was designed in OnShape and printed with orange PLA filament. Figure 7 shows the original design with two wooden $1 \frac{1}{2}$ inch dowels, which were later changed to a single dowel design to reduce weight. To maintain a distance of 10 inches between the 360-degree camera and the payload, we cut the dowels to an appropriate length. The dowels were then attached to a laser-cut piece of plywood. The top of the selfie stick was attached to the top of the payload, where a PETG ring supported it. This ring also allowed for a tight fit between the insulation foam and the rocket body tube. 


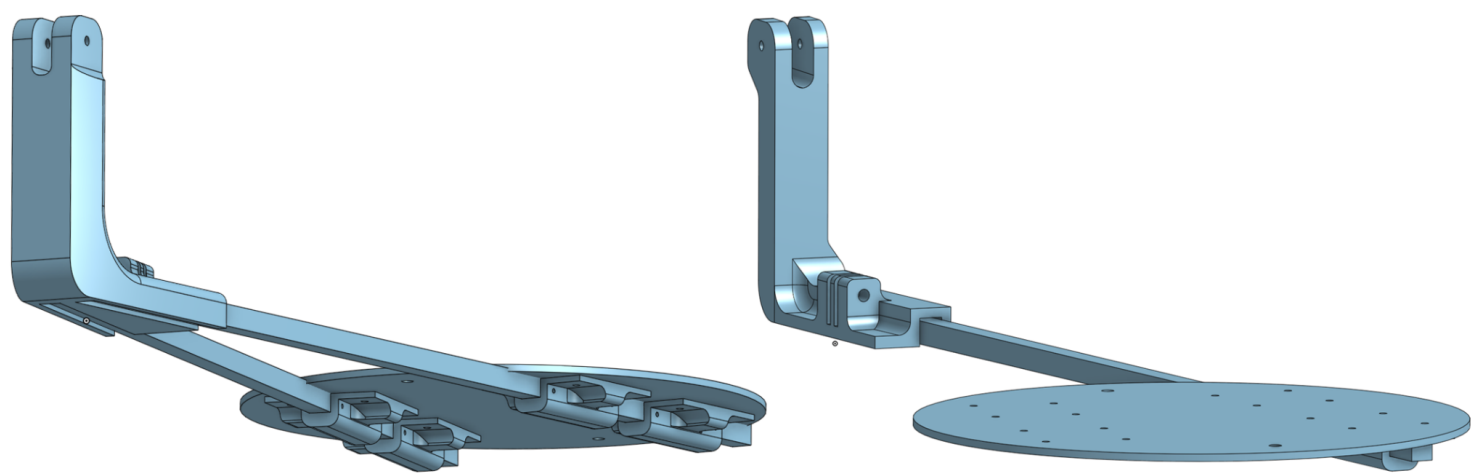

Figure 7. Initial selfie stick with two dowels and final selfie stick with single dowel.

Results Both cameras remained powered throughout the flight and recorded footage from the launch to the landing. Figures 8 and 9 depict images captured from both cameras during the flight. This result was unexpected for the 360-degree camera, which would overheat and turn off after about an hour of testing indoors. However, the camera performed appropriately during the flight due to the high altitudes and wind keeping it cool.

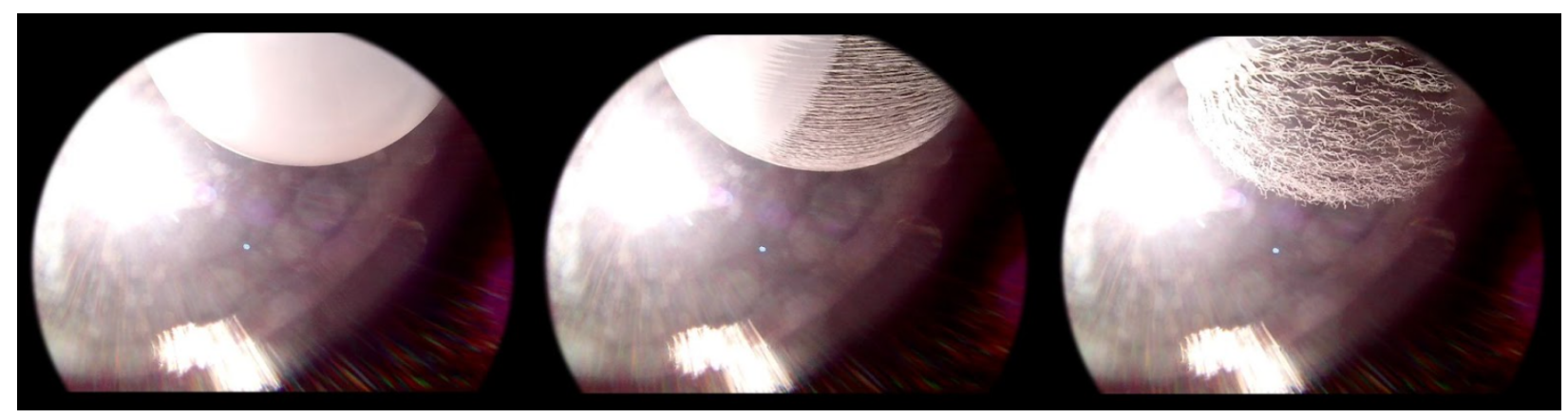

Figure 8: Full spectrum camera balloon images at maximum altitude.

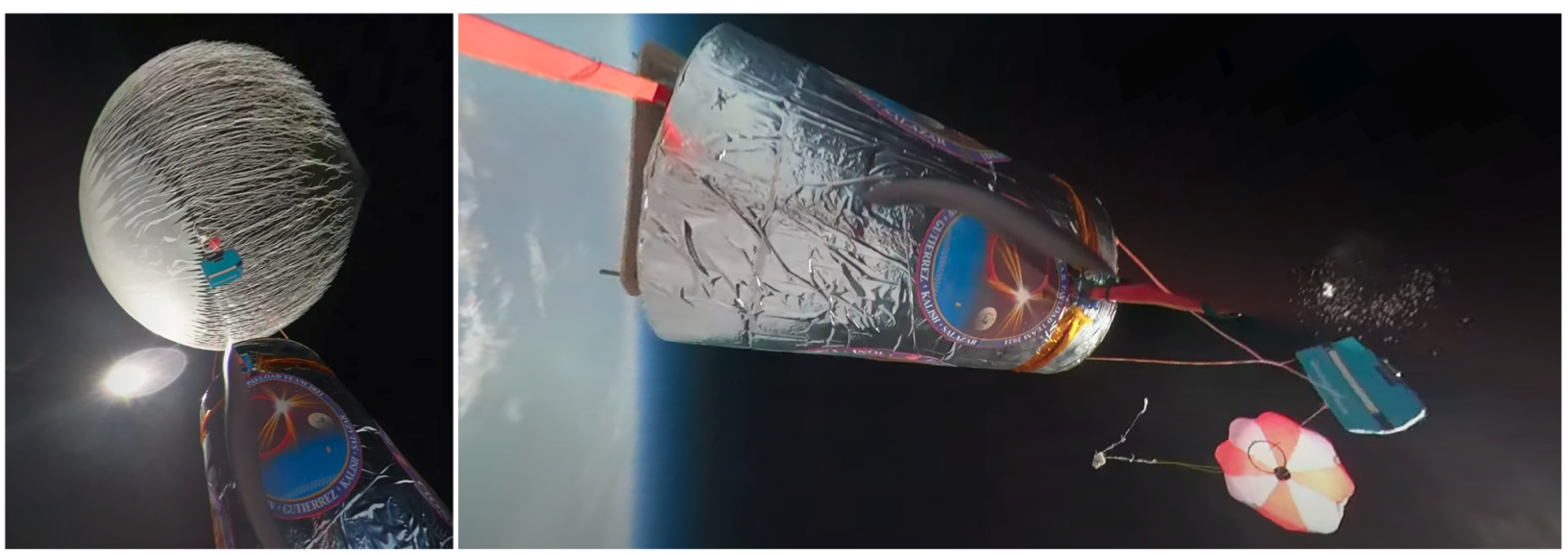

Figure 9. 360 degree camera bursting balloon images at maximum altitude.

The selfie stick remained intact upon landing, keeping the 360-degree camera secure. The 360-degree videos were processed successfully using GoPro Studio with a Macbook Pro. 
Using a laptop PC proved to be unreliable with constant crashes. Unfortunately, the launch day temperatures varied from $90^{\circ} \mathrm{F}$ to $100^{\circ} \mathrm{F}$, and shortly after the payload was ready to launch, the filter mechanism's friction fit connection with the servo motor failed. This outcome was unexpected, as the filtering mechanism had been tested for over six hours, indoors and outdoors. Due to this failure, the only usable data involving the filtration mechanism came from preliminary testing before the launch. Figure 10 shows images from the full spectrum camera with three different filters. However, the full spectrum camera did capture the balloon at maximum altitude, which confirms that the 45-degree angle was a good starting place. The top third of the frame showed the balloon at its maximum radius.

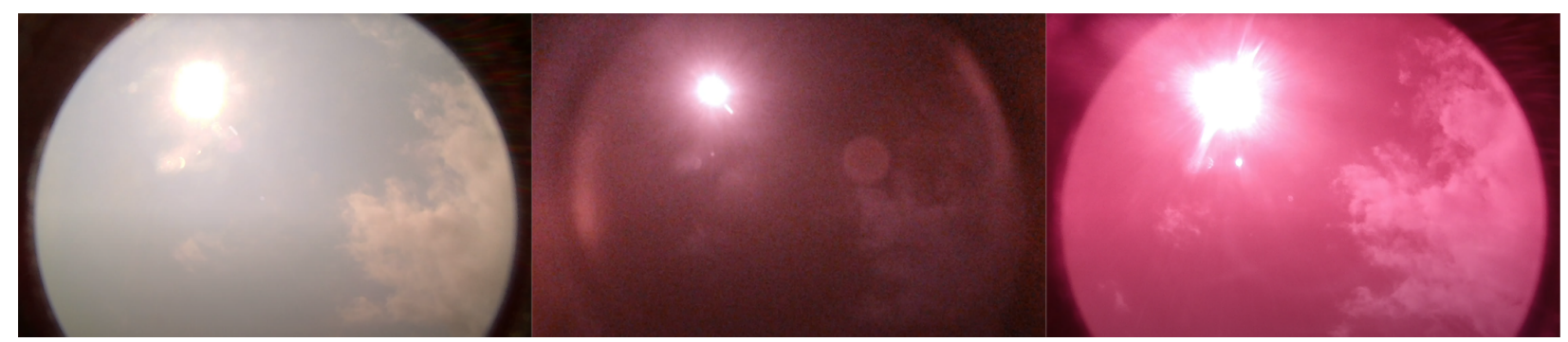

Figure 10. Full spectrum camera filtered images: IR cut-off, Solar, IR.

Conclusion The implementation of the 360-degree camera with the selfie stick was a success. It proved to be sturdy enough to survive the flight and landing. In addition, it provided excellent videos that include the launch, balloon burst, and landing of the payload. The full spectrum camera performed well while being protected by the 3D-printed enclosure. The filter mechanism was a good start, with much room for refinement. Future teams should explore alternative options to the heat-sensitive PLA filament used like ABS plastic and wood. Weight savings are possible by diminishing the size of the extended battery bank and using lighter 360-degree cameras. These options will likely come at a higher cost.

\section{Cosmic Radiation Shielding}

The objective of the cosmic radiation shielding project was to determine how effective low-density polyethylene (LDPE) is in shielding galactic cosmic radiation (GCR).

Introduction As the demand for space travel and colonization increases, it is vital to ensure that astronauts are safe during extended periods in space. The magnetic field surrounding Earth provides living beings with protection from galactic cosmic radiation or highly charged ions that travel through the galaxy at the speed of light. Typical spacecraft walls or human skin are ineffective in preventing these particles from passing through (Dunbar, Perez, 2019). Long-term exposure to ionizing radiation can result in damage to DNA, tissues, and organs. Additionally, this exposure can cause life-threatening conditions such as cancer to develop (National Institute of Environmental Health Sciences, 2021). There is potential to explore ways to shield from galactic cosmic radiation through a high altitude balloon because Earth's magnetic field weakens at higher altitudes. During the 2024 total solar eclipse flight, the team may develop a relationship between the light intensity and the magnitude of GCR. 
Methods To effectively shield from GCR, the team considered that hydrogen atoms stabilize highly charged particles characteristic of GCR. Polyethylene was a viable option due to its high concentration of hydrogen atoms. Additionally, previous research supported the ability of polyethylene to shield from GCR. Thicker polyethylene shields proved to be more successful in blocking radiation (Narici, Casolino, Di Fino, et al., 2017). Due to weight and size constraints, the team decided that half-inch thick LDPE was the most appropriate shielding thickness to test on the payload. The team selected LDPE for its flexibility and availability from local suppliers.

Utilizing two separate Geiger counters (detects ionizing radiation), custom LDPE shielding, an Arduino Uno, an SD card shield (records data from components on SD card), and an RTC (records time) gave the team all data necessary in evaluating the shielding capabilities of LDPE. Each part described can be seen in Figure 11.
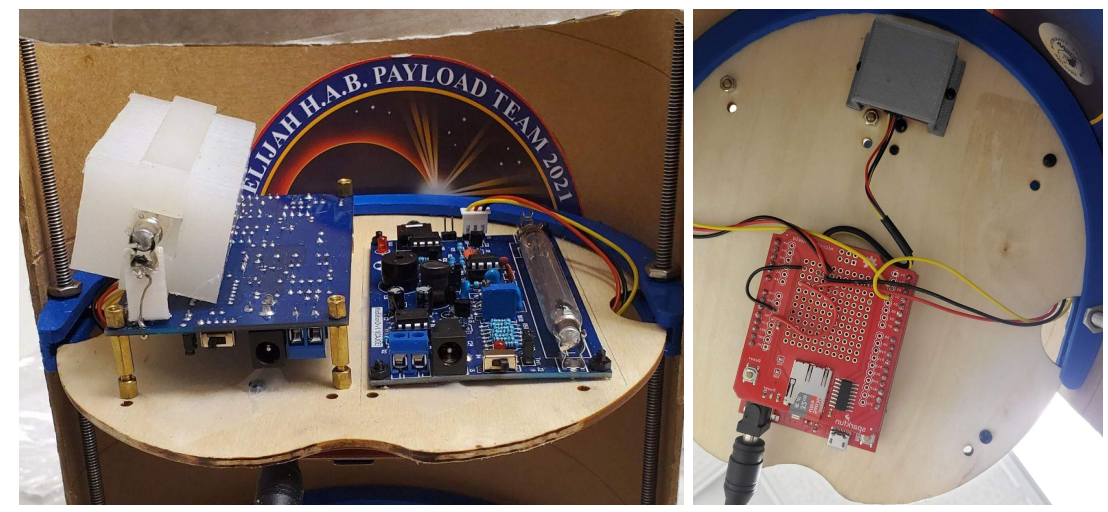

Figure 11. GCR Shielding Experimental Design

Results The graphs seen below in Figure 12 and Figure 13 show the data captured on the SD card during the flight plotted in several ways to analyze the effectiveness of the LDPE shielding. Figure 12 depicts the counts per minute experienced by the exposed and shielded Geiger counters during the flight. Another method of analysis involved dividing the shielded CPM by the exposed CPM, as shown in Figure 13. The vertical green line present in both figures represents when the balloon shredded and began its descent. 


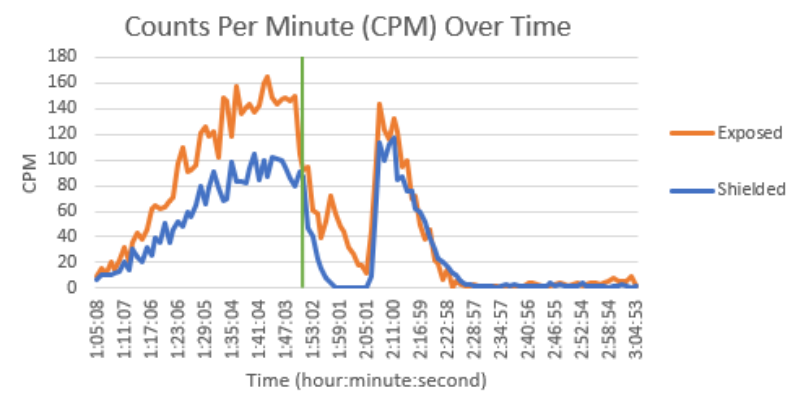

Figure 12. Plot of Counts Per Minute Over Time

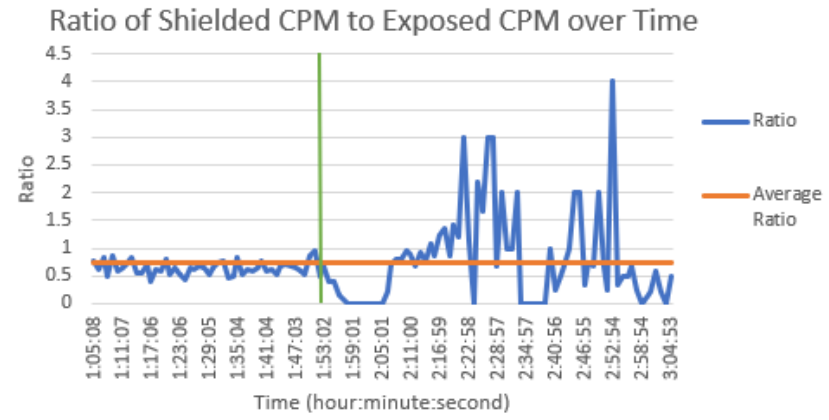

Figure 13. Plot of Shielded Counts Per Minute to Exposed Counts Per Minute Over Time

Conclusion The average ratio of shielded CPM to exposed CPM over the flight was 0.73 , indicating that LDPE reduced the radiation that the shielded Geiger counter tube experienced overall. A value of 1.00 implies that LDPE did not protect from radiation, while a ratio greater than 1.00 means that LDPE increased radiation exposure. During the balloon's descent, the amount of shielded CPM to exposed CPM reached a peak of 4.0. This ratio is significantly higher than the typical ratio experienced during the balloon's ascent. This result may be due to a lack of GCR within the earth's magnetosphere or the exposed Geiger counter tube coming loose.

\section{Interstellar Radiation Environment}

Introduction The rocket equation applies to all space destined vehicles. This equation restricts a spacecraft's total change in velocity based on the chosen fuel's chemical potential energy and the initial to final mass ratio. A spacecraft that uses conventional propellant-based acceleration cannot carry sufficient fuel to escape orbit while achieving a great enough velocity to reach nearby interstellar destinations in a reasonable time.

Circumventing complete reliance on propellants, solar sails, which utilize an alternative means of acceleration after entering orbit, have been successfully demonstrated on numerous occasions (Spencer et. al., 2019). By exploiting momentum transferred from reflected photons, solar sails can accelerate to relativistic speeds without resource limitations. Yet, this technology is not without limitations. The functional lifetime of the reflective sail material, which enables the characteristic, long-term, continual acceleration of a solar sail, is strongly limited by its exposure to environmental radiation (Edwards et. al., 2003).

At this time, forming a predictive model of the local radiation environment is an active area of research. Some limitations to obtaining a mathematical model are low-intensity interactions with GCRs and a general lack of observations (Fu et. al., 2020). Alternatively, several empirical models exist. They originate back to data from the Advanced Composition Explorer (ACE) satellite (SR-NIEL, 2020).

Supporting the next generation of space exploration, the radiation environment project is 
an ongoing effort to study the radiation landscape between our star system and its interstellar neighbors. This experiment seeks to investigate the validity of radiation environment models created with ACE data. In addition, observations can refine current models by cross-referencing with existing ACE data to gain greater insight into sources of error such as magnetic fields, solar proximity, and obstructive debris.

Methods To observe galactic cosmic rays originating from nearby solar systems, the team decided to construct a scintillator and silicon-photomultiplier (SiPM) particle detector. The reason for choosing this design is its ability to record GCR energy, fluence, and particle identity (Figure 14). Additionally, the low power requirements, single-photon accuracy, and affordability of SiPMs make them a preferable alternative to the photomultiplier tubes traditionally used in similar applications.

The scintillating material selected for the detector was an organic, polyvinyl toluene crystal. The characteristics of this material are its sensitivity to charged particles, as well as alpha, beta, and high energy gamma radiation (Saint-Gobain Ceramics and Plastics, 2018). To further support the material's use on board a high altitude balloon, the chosen scintillator maintained reliability in the presence of moisture and low temperatures experienced in the stratosphere.

To capture event data, the team decided to purchase a KETEK PM3335-WL SiPM. This SiPM possesses a wavelength sensitivity range from $300 \mathrm{~nm}$ to $900 \mathrm{~nm}$, with peak sensitivity occurring at $430 \mathrm{~nm}$ (KETEK, 2018). Upon each GCR interaction, the selected scintillator crystal emits photons in wavelengths ranging from $360 \mathrm{~nm}$ to $520 \mathrm{~nm}$, peaking at $425 \mathrm{~nm}$ (Saint-Gobain Ceramics and Plastics, 2018). With a sensitivity range overlapping that of emitted photons, and a peak sensitivity close to the scintillator's peak emission wavelength, the PM3335-WL provides the highest achievable detection efficiency within budget constraints.

Due to the high cost of off-the-shelf scintillator set-ups, the team decided to construct the supporting circuitry. Circuit schematics from physicsopenlab.org dramatically simplified the design process. The open-source plans served as the design foundation, but some modifications were necessary to support KETEK SiPMs, SD card interfacing, and power supplies shared among payload experiments.

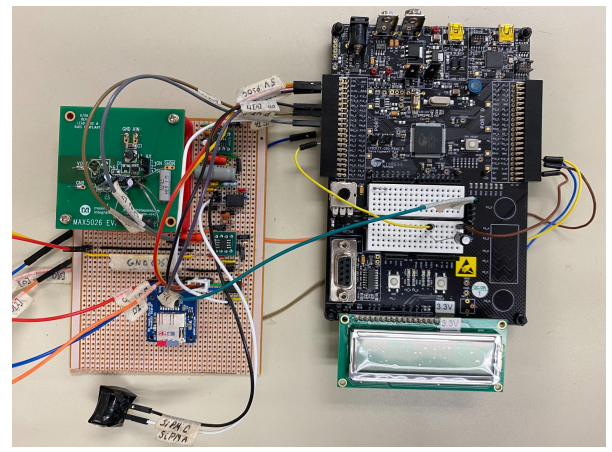

Figure 14. Silicon Photomultiplier Circuit Design 
Results Shortly before launching the balloon, an op-amp in the peak detect and hold circuit failed; thus, rendering the detector ineffective. Without suitable replacements and insufficient time to order one, the experiment was removed from the payload. As such, this project did not yield any experimental results.

Conclusion Despite being unable to perform this experiment, efforts to observe GCRs originating from nearby solar systems will continue. To fully understand interstellar radiation, it is necessary to obtain a wealth of data. The appropriate amount of data needed exceeds that which can be collected during the short duration of a balloon flight. To that end, long duration observation opportunities will be pursued.

Additionally, this project has provided insights that will benefit future Elijah HAB teams. Most notably, the team found an effective, low-cost method of observing GCR fluence and energy. Elijah HAB scholars who wish to incorporate these functionalities into future GCR studies may do so without designing and programming experimental hardware. Also, a cautionary tale, emphasizing the importance of preparing for unforeseen events such as hardware failures, has been written.

\section{General Conclusion}

Methods and results from this project should be studied and improved upon by future teams to determine which variables to observe further in preparation for the 2024 total solar eclipse.

\section{Acknowledgments}

Dr. William Farrow

Vivian Mickelson

Richard Phillips

Luis A. Rodriguez

Justin Sommer

\section{References}

Altomare, C., De La Torre Luque, P., Di Venere, L., Fusco, P., Gargano, F., Giordano, F., Hu, P., Loparco, F., Loporchio, S., Mazziotta, M. N., \& Serini, D. (2020). Particle identification capability of Plastic scintillator tiles equipped with SiPMs for the High Energy cosmic-Radiation Detection (HERD) facility. Nuclear Instruments And Methods In Physics Research, 983, 164476. https://doi.org/10.1016/j.nima.2020.164476

Fu, S., Zhao, L., Zank, G., Wang, M., \& Jiang, Y. (2020). An ACE/CRIS-observation-based galactic cosmic rays heavy nuclei spectra model II. Science China Physics, Mechanics \& Astronomy, 63, 219511. https://doi.org/10.1007/s11433-019-9423-3

KETEK GmbH. (2020, January). KETEK PM33xx WL AO Datasheet. https://www.ketek.net/wp-content/uploads/KETEK-PM33xx-WL-A0-Datasheet.pdf

KETEK GmbH. (2018, April 25). SiPM $\quad$ FAQ. 
https://www.ketek.net/documentation/sipm-faq/

Narici, L., Casolino, M., Di Fino, L., Larosa, M., Picozza, P., Rizzo, A., \& Zaconte, V. (2017, May 10). Performances of kevlar and Polyethylene as radiation shielding on-board the International space station in high LATITUDE radiation environment. Nature News. https://www.nature.com/articles/s41598-017-01707-2\#Tab3.

Perez, J. (2017, April 13). Why space radiation matters. NASA. https://www.nasa.gov/analogs/nsrl/why-space-radiation-matters.

Pettit, D. (2012, May 1). The tyranny of the rocket equation. National Aeronautics and Space

Administration(NASA).https://www.nasa.gov/mission_pages/station/expeditions/expediti on30/tryanny.html

Physics Open Lab. (2017, November 28). Front-end electronics for SiPM. https://physicsopenlab.org/2017/11/28/front-end-electronics-for-sipm/

Physics Open Lab. (2017, November 28). Programmable power supply for SiPM bias. https://physicsopenlab.org/2017/11/28/programmable-power-suppy-for-sipm-bias/

Physics Open Lab. (2017, November 28). PSoC controlled SiPM detector. https://physicsopenlab.org/2017/11/28/psoc-controlled-sipm-detector/

Saint-Gobain Ceramics \& Plastics, Inc. (2018, February). BC-400, BC-404, BC-408, BC-412, BC-416 Premium Plastic Scintillators. https://www.crystals.saint-gobain.com/sites/imdf.crystals.com/files/documents/bc400-40 4-408-412-416-data-sheet.pdf

Screen Relativistic Non-ionizing Energy Losses (SR-NIEL). (2020, December 14). LET distributions for Fe-ions from SPENVIS, CREME and SR-NIEL/HelMod frameworks: dependence of the estimated number of SEE on solar modulation model. SR-NIEL. http://www.sr-niel.org/index.php/sr-niel-long-write-up/let-distributions-for-fe-ions-from-s penvis-creme-and-sr-niel-helmod

Spencer, D. A., Johnson, L., \& Long, A. C. (2019). Solar sailing technology challenges. Aerospace Science And Technology, 93, 105276. https://doi.org/10.1016/j.2019.07.009

U.S. National Library of Medicine. (2021, May 28). Radiation exposure. MedlinePlus. https://medlineplus.gov/radiationexposure.html. 